\title{
Comparison and Appraisal of Approximation Formulas for Total Elastic Molecular Scattering Cross Sections*
}

\author{
R. B. BERNSTEIN AND K. H. KRAMER \\ Chemistry Departments, University of Michigan, Ann Arbor, Michigan, and University of Wisconsin, Madison, Wisconsin
}

(Received 7 January 1963)

\begin{abstract}
The Massey-Mohr (MM), Schiff (S), and Landau-Lifshitz (LL) approximations for the total elastic cross section $(Q)$ are intercompared. All can be shown to follow from the same assumption, (i.e., the classical small-angle deflection function, thence the Jeffreys-Born phases via the semiclassical equivalence relationship), sufficing to determine the velocity dependence of $Q$. Thus, for $V= \pm C^{(s)} / r^{8}, Q^{(s)}=p(s)\left[C^{(s)} / \hbar v\right]^{2 /(8-1)}$ The coefficient $p(s)$ is the same for the $\mathrm{S}$ and $\mathrm{LL}$ approximations; the ratio $p_{\mathrm{SLL}}(s) / p_{\mathrm{MM}}(s) \geq 1,(<1.075)$; it is 1.0709 and 1.0458 for $s=6$ and 12 , respectively.

A numerical calculation for a repulsive $(s=12)$ interaction shows that the SLL formula reproduces the partial-wave calculated $Q$ to within $\frac{1}{3} \%$. A graphical presentation suggests the generality of this result; it also indicates the source of bias in the MM approximation. For a "realistic" intermolecular potential, (restricting consideration to collisions in the "thermal" energy range), the influence of the repulsion is only to produce undulations in $Q(v)$; the correct value of $C^{(6)}$ may be obtained by velocity averaging the "apparent" $C_{\text {BLL }}{ }^{(6)}$.
\end{abstract}

\section{PREVIOUS WORK}

$\mathbf{T}$ HE Massey-Mohr approximation formula ${ }^{1}$ has often been used to interpret total elastic cross sections in terms of the long-range attractive part of the intermolecular potential

$$
V(r)=-C^{(s)} / r^{s}
$$

the MM formula is

$$
Q_{M M}^{(s)}(v)=p_{M M}(s)\left[C^{(s)} / \hbar v\right]^{2 /(s-1)},
$$

where $v$ is the relative velocity and $p_{M M}(s)$ is a known numerical coefficient (see Table I). Thus the desired potential constant may be directly calculated from the cross section. ${ }^{2-4}$

Recent experiments with velocity-selected ${ }^{5-7}$ and Maxwellian ${ }^{4}$ molecular beams have tended to confirm the MM velocity dependence; i.e., the derived values of $C^{(6)}$ are found to be essentially constant over a wide range of collision energies. ${ }^{8}$ In addition, the relative values of $C^{(6)}$ obtained for different gas pairs are well

* Financial support of this work by the U. S. Atomic Energy Commission, Division of Research is gratefully acknowledged.

${ }^{1}$ H. S. W. Massey and C. B. O. Mohr, Proc. Roy. Soc. (London) A144, 188 (1934).

${ }^{2}$ H. S. W. Massey and R. A. Buckingham, Nature 138, 77 (1936).

${ }^{3}$ E. W. Rothe and R. B. Bernstein, J. Chem. Phys. 31, 1619 (1959).

${ }^{4}$ H. Schumacher, R. B. Bernstein, and E. W. Rothe, J. Chem. Phys. 33, 584 (1960).

${ }^{5}$ H. Pauly, Z. Naturforsch. 15a, 277 (1960).

${ }^{6}$ R. C. Schoonmaker, J. Phys. Chem. 65, 892 (1961).

${ }^{7}$ E. W. Rothe, P. K. Rol, S. M. Trujillo, and R. H. Neynaber, Phys. Rev. 128, 659 (1962).

8 Deviations have been observed for systems in which dipoledipole forces play a role ${ }^{4}$ and for systems involving the lighter atoms.7,9-11. For the latter case the deviations are satisfactorily explained in a semiquantitative way..$^{12,13}$

${ }^{9}$ H. U. Hostettler and R. B. Bernstein, Phys. Rev. Letters 5, $318(1960)$.

${ }^{10}$ H. Harrison, J. Chem. Phys. 37, 1164 (1962).

${ }_{11}$ P. K. Rol and E. W. Rothe, Phys. Rev. Letters 9, 494 (1962).

12 R. B. Bernstein, J. Chem. Phys. 37, 1880 (1962).

${ }^{13}$ R. B. Bernstein, J. Chem. Phys. 38, (1963) 38, 515 (1963). correlated ${ }^{2,3,14,15}$ by the standard dispersion-force theory. However, absolute values of $C$ thus obtained are subject to uncertainty due to the unknown inaccuracy of the approximation when applied either to the monotonic potential for which it is appropriate or to a "realistic" intermolecular potential (i.e., with shortrange repulsion).

Calculations, ${ }^{16}$ based on a complete phase shift analysis for a L-J $(12,6)$ potential showed (for a specific example) that the "true" cross section $Q^{(12,6)}(v)$ oscillated around an "average" value, $\left\langle Q^{(12,6)}(v)\right\rangle$, which was greater than $Q_{M M}{ }^{(6)}$ by about $10 \%$. Helbing and Pauly ${ }^{17}$ reported partial-wave calculations of $Q^{(12,6)}(v)$ for another example; the results exceeded $Q_{\mathrm{MM}^{(6)}}(v)$ by about $7 \%$. Similar partial-wave computations ${ }^{18}$ have recently been carried out with a wide parameter variation. It was noted that $\left\langle Q^{(2,6)}(v)\right\rangle$ was greater than $Q_{M M}{ }^{(6)}(v)$ by $7.5 \pm 1.5 \%$. A theoretical analysis ${ }^{19}$ of the extrema in the velocity dependence of $Q^{(12,6)}$ in terms of the influence of the repulsive phases has shown that $\left\langle Q^{(12,6)}(v)\right\rangle$ should be identical to $Q^{(6)}(v)$ in the "thermal" or "low-velocity" region. The above results imply a velocity-independent bias in the MM approximation (even when applied to a monotonic potential).

An alternate approximation formula ${ }^{20}$ for $Q^{(s)}$ is that of Landau and Lifshitz. ${ }^{21}$ The LL formula is identical

\footnotetext{
${ }^{14}$ For a review, see H. Pauly, Fortschr. Physik 9, 613 (1961).
}

${ }^{15}$ E. W. Rothe, L. L. Marino, R. H. Neynaber, P. K. Rol, and S. M. Trujillo, Phys. Rev. 126, 598 (1962).

${ }^{16}$ R. B. Bernstein, J. Chem. Phys. 33, 795 (1960); 34, 361 (1961).

${ }^{17}$ R. Helbing and H. Pauly, Diplomarbeit (Helbing), University of Bonn, 1961.

${ }^{18}$ E. W. Rothe, P. K. Rol, and R. B. Bernstein, Phys. Rev. 129, (1963) (in press).

${ }^{19}$ R. B. Bernstein, "Semiclassical Analysis of the Extrema in the Velocity Dependence of Total Elastic Scattering Cross Sections: Relation to the Bound States," J. Chem. Phys. (to be published).

${ }^{20}$ Used, for example, by E. A. Mason (private communication, 1962).

${ }^{21}$ L. D. Landau and E. M. Lifshitz, Quantum Mechanics (Pergamon Press, Ltd., London, 1959), p. 416. 
TABLE I. Numerical coefficients in the approximation formulas.

\begin{tabular}{rrr}
\hline$s$ & $p_{\mathrm{MM}}(s)$ & $p_{\mathrm{SLL}}(s)$ \\
\hline 3 & 18.849 & 19.739 \\
4 & 10.613 & 11.373 \\
5 & 8.464 & 9.093 \\
6 & 7.547 & 8.083 \\
7 & 7.062 & 7.529 \\
8 & 6.771 & 7.185 \\
9 & 6.583 & 6.956 \\
10 & 6.454 & 6.793 \\
11 & 6.363 & 6.674 \\
12 & 6.296 & 6.584 \\
13 & 6.246 & 6.514 \\
14 & 6.207 & 6.458 \\
15 & 6.178 & 6.413 \\
16 & 6.154 & 6.376 \\
24 & 6.080 & 6.233 \\
\hline
\end{tabular}

to Eq. (2) with another value for the coefficient, i.e., $p_{\mathrm{LL}}(s)$. Still another approximation formula ${ }^{22}$ is that of Schiff. ${ }^{23}$ For a central potential the Schiff formula reduces to the form of $\mathrm{Eq}$. (2), with coefficient $p_{S}(s)$.

In the following section the three approximations are intercomposed. In the subsequent section a numericalgraphical illustration is presented for the case of a pure repulsive interaction with $s=12$.

\section{INTERCOMPARISON OF APPROXIMATION TREATMENTS}

The standard expression for the cross section:

$$
Q(k)=\left(8 \pi / k^{2}\right) \sum_{l}\left(l+\frac{1}{2}\right) \sin ^{2} \eta_{l}(k)
$$

requires a knowledge of all the phase shifts $\eta_{l}(k)$. For a potential of the form of Eq. (1), the classical smallangle deflection function ${ }^{24}$ can be expressed;

$$
\theta=-\left[(s-1) f(s) C^{(s)} / E b^{s}\right],
$$

where $E=\frac{1}{2} \mu v^{2}, b$ is the impact parameter, and $f(s)=$ $\frac{1}{2}(\pi)^{\frac{1}{3}} \Gamma\left(\frac{1}{2} s-\frac{1}{2}\right) / \Gamma\left(\frac{1}{2} s\right)$. Integrating via the semiclassical equivalence relationship, ${ }^{25}$ making the usual substi-

22 First applied to the molecular scattering problem by C. Schlier; see, for example, K. Berkling, R. Helbing, K. Kramer, H. Pauly, Ch. Schlier, and P. Toschek, Z. Physik. 166, 406 (1962); see also references 14 and 17.

${ }_{23}$ L. I. Schiff, Phys. Rev. 103, 443 (1956).

${ }^{24}$ E. H. Kennard, Kinetic Theory of Gases (McGraw-Hill Book Company, Inc., New York, 1938).

${ }_{25}$ R. B. Bernstein, J. Chem. Phys. 36, 1403 (1962). Erratum: Eq. (7b) should read

$$
\eta_{l}=\frac{3 \pi}{32} \frac{2 \mu C^{(6)}}{\hbar^{2}} \frac{k^{4}}{\left(l+\frac{1}{2}\right)^{6}} ;
$$

see reference 13 for list of other errata. tutions $\left[b=\left(l+\frac{1}{2}\right) / k ; E=\hbar^{2} k^{2} / 2 \mu\right], \mathrm{Eq}$. (4) yields the phase shift (valid in the limit of high $l$ and/or small $\eta_{l}$ ):

$$
\eta_{l}=\left(\mu C^{(s)} / \hbar^{2}\right)\left[k^{s-2} /\left(l+\frac{1}{2}\right)^{s-1}\right] f(s) .
$$

MM and LL employ the Jeffreys-Born (JB) approximation

$$
\begin{aligned}
\left(\eta_{l}\right)_{\mathrm{JB}}=\frac{\mu C^{(s)}}{\hbar^{2}} \int_{\left(l+\frac{1}{2}\right) / k}^{\infty} & \frac{d r}{r^{s}\left\{k^{2}-\left[\left(l+\frac{1}{2}\right) / r\right]^{2}\right\}^{\frac{1}{2}}} \\
& =\frac{\mu C^{(s)}}{k \hbar^{2}} \int_{b}^{\infty} \frac{d r}{r^{s-1}\left[r^{2}-b^{2}\right]^{\frac{1}{2}}}=\left(\eta_{b}\right)_{\mathrm{JB}},
\end{aligned}
$$

which yields a result identical with Eq. (5) (note that LL replace $l+\frac{1}{2}$ by $l$ ); whereas MM make use of the approximation only for the higher-order phases for which it is valid [i.e., $l>L$, where $\eta_{L}(k)=\frac{1}{2}$ ], and $L L$ employ it throughout. LL replace the sum [Eq. (3)] by an integral:

$$
Q_{\mathrm{LL}}^{(s)}=\frac{8 \pi}{k^{2}} \int_{0}^{\infty} l \sin ^{2}\left(\eta_{l}\right)_{J \mathrm{~B}} d l .
$$

They note that the principal contribution to the integral arises from the high-order phases in any case. The LL result is of the form of Eq. (2) with a coefficient given by

$$
\begin{aligned}
& p_{\mathrm{LL}}(s)=2 \pi^{s /(s-1)} \sin \left[\frac{\pi}{2} \frac{s-3}{s-1}\right] \Gamma\left(\frac{s-3}{s-1}\right) \\
& \times\left[\frac{\Gamma[(s / 2)-(1 / 2)]}{\Gamma(s / 2)}\right]^{2 /(s-1)} \quad(\text { valid for } s>3) .
\end{aligned}
$$

An alternate evaluation of the integral $[\mathrm{Eq}$. (6) $]$ can be carried out, yielding a somewhat preferable form, valid for $s>2$.

$p_{\mathbf{L L}}(s)=\frac{2^{2 /(s-1)} \pi^{2}}{\sin [\pi /(s-1)] \Gamma[2 /(s-1)]}[f(s)]^{2 /(s-1)}$.

[These forms are interconvertible for $s>3$, by making use of the relation $\Gamma(x) \Gamma(1-x)=\pi / \sin x \pi]$.

MM introduce the random-phase approximation for the large, low-order phases $(l<L)$. The sum (3) is broken into two parts: Region I (for $l<L$ ), in which $\sin ^{2} \eta_{l}$ is replaced by $\left\langle\sin ^{2} \eta_{l}\right\rangle=\frac{1}{2}$, and II (for $l \geq L$ ), where $\sin \eta_{l}$ may be approximated by $\left(\eta_{l}\right)_{\mathrm{JB}}$ and the summation replaced by integration. The fractional contribution of the JB region (II) to $Q_{M M}$ is found to be only $1 /(2 s-3)$. The final formula for $Q_{M M}$ is $E q$. (2), with

$$
p_{M M}(s)=2^{2 /(s-1)} \pi[(2 s-3) /(s-2)][f(s)]^{2 /(s-1)} .
$$

The $S$ approximation [for a spherically symmetric potential of the form of Eq. (1)] may be written:

$$
Q_{\mathrm{S}}^{(s)}=4 \int_{-\infty}^{\infty} \int_{-\infty}^{\infty} \sin ^{2} \gamma_{b} d x d y
$$


FIG. 1. Plot of $p_{\mathrm{SLL}} / p_{\mathrm{MM}}$ vs $s$.

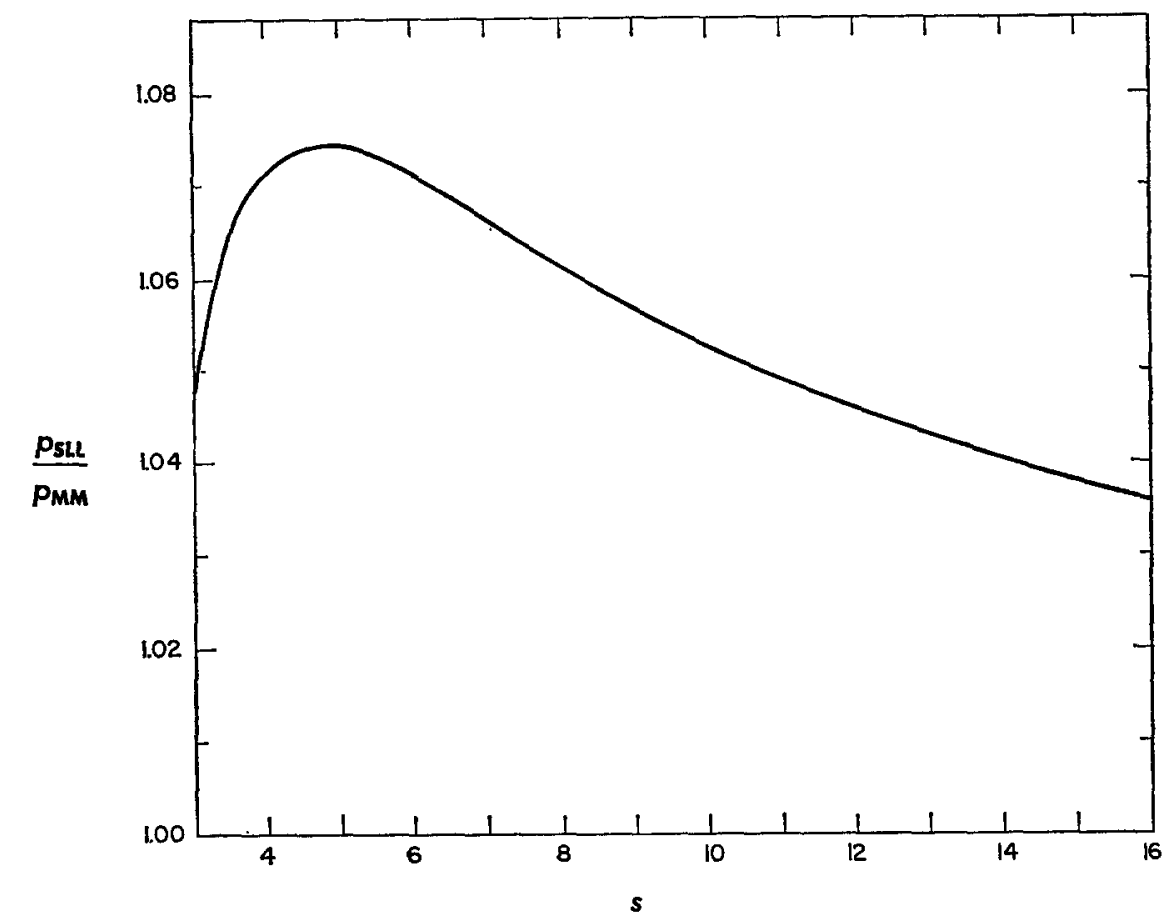

where

$$
\gamma_{b} \equiv\left(-\mu C^{(s)} / 2 k \hbar^{2}\right) \int_{-\infty}^{\infty} r^{-s} d z
$$

Transforming to cylindrical coordinates $(z, \phi, b)$ and integrating over $\phi$, Eq. (9) yields

$$
Q_{\mathrm{S}}^{(s)}=8 \pi \int_{0}^{\infty} \sin ^{2} \gamma_{b} b d b=\frac{8 \pi}{k^{2}} \int_{0}^{\infty}\left(l+\frac{1}{2}\right) \sin ^{2} \gamma_{b} d l
$$

Using the small angle approximation $r^{2}=b^{2}+z^{2}$, (i.e., $r d r=z d z$ at constant $b$ ), Eq. (10) yields

$$
\gamma_{b}=\frac{-\mu C^{(s)}}{2 k \hbar^{2}} \cdot\left[2 \cdot \int_{b}^{\infty} \frac{r d r}{r^{s}\left(r^{2}-b^{2}\right)^{\frac{1}{2}}}\right]=-\left(\eta_{b}\right)_{\mathrm{JB}}
$$

so that $-\gamma_{b}$ is identified with the JB phase, obtainable as before from the classical small-angle deflection function. Thus $Q_{\mathbb{S}^{(s)}}[\mathrm{Eq} .(11)]$ is identical with $Q_{\mathrm{LL}}{ }^{(s)}[\mathrm{Eq}$. $\left.\left(3^{\prime}\right)\right]$, i.e., $p_{S}(s)=p_{\mathrm{LL}}(s)=p_{\mathrm{SLL}}(s)$ (see Table I and Fig. 1).

\section{NUMERICAL AND GRAPHICAL ILLUSTRATION}

For the purpose of illustrating and comparing the approximation methods, a specific example is shown for a pure repulsive potential, $V=C / r^{12}$. A repulsive potential ensures a proper inner boundary condition for the radial wavefunction, ${ }^{16}$ always producing a finite (negative) $s$-wave phase shift, obviating the need for introducing any spurious "core" in the potential. Tabulated values of the classical deflection function for this potential are available 26 ; thus reduced phases and

${ }^{26} \mathrm{~J}$. O. Hirschfelder, C. F. Curtiss, and R. B. Bird, Molecular Theory of Gases and Liquids (John Wiley \& Sons, Inc., New York, 1954). phases can readily be calculated, ${ }^{25}$ so that a "true" value of the cross section $Q_{0}$ can be computed. In order for the example to satisfy the semiclassical requirement of a statistically large number of phases, the velocity parameter must exceed some minimum value; for the present illustration it was such that over 100 partial waves were involved.

The following equations define the problem and give the particular conditions of the example. The symbols are those of references 16,25 , and 26.

Here

$$
\begin{aligned}
& V=4 \epsilon \sigma^{12} / r^{12} ; \quad A=k \sigma=\mu \nu \sigma / \hbar=69.282, \\
& B=2 \mu \epsilon \sigma^{2} / \hbar^{2}=100.0, \quad K=E_{0} / \epsilon=A^{2} / B ;
\end{aligned}
$$

as usual,

$$
\begin{aligned}
Q_{0}{ }^{*}=Q_{0} / \pi \sigma^{2} & =\left(8 / A^{2}\right) \cdot \sum_{l}\left(l+\frac{1}{2}\right) \sin ^{2} \eta_{l}(A) \\
& =\left(8 / A^{2}\right) \cdot \sum_{l} q^{(l)}(A)
\end{aligned}
$$

To obtain the phases

$$
\eta^{*}\left(b^{*}, K\right)=-\frac{1}{2} \cdot \int_{b^{*}}^{\infty} \theta d b^{*}=-\frac{1}{2}\left(\frac{48}{K}\right)^{1 / 12} \cdot \int_{y_{0}}^{\infty} x d y_{0}
$$

with $y_{0}=b^{*}\left(\frac{1}{48} K\right)^{1 / 12}$, where $b^{*}=b / \sigma$.

Then $\eta_{l}(A)=A \eta^{*}\left(b^{*}, K\right)$, with $l=A b^{*}-\frac{1}{2}$.

The integrals, Eq. (14), were evaluated graphically for $0 \leq y_{0} \leq 1.5$; for $y_{0}>1.5$, the small-angle approximation for the deflection function was used:

$$
\chi(=\theta)=\left(693 \pi / 6144 y_{0}{ }^{12}\right),
$$




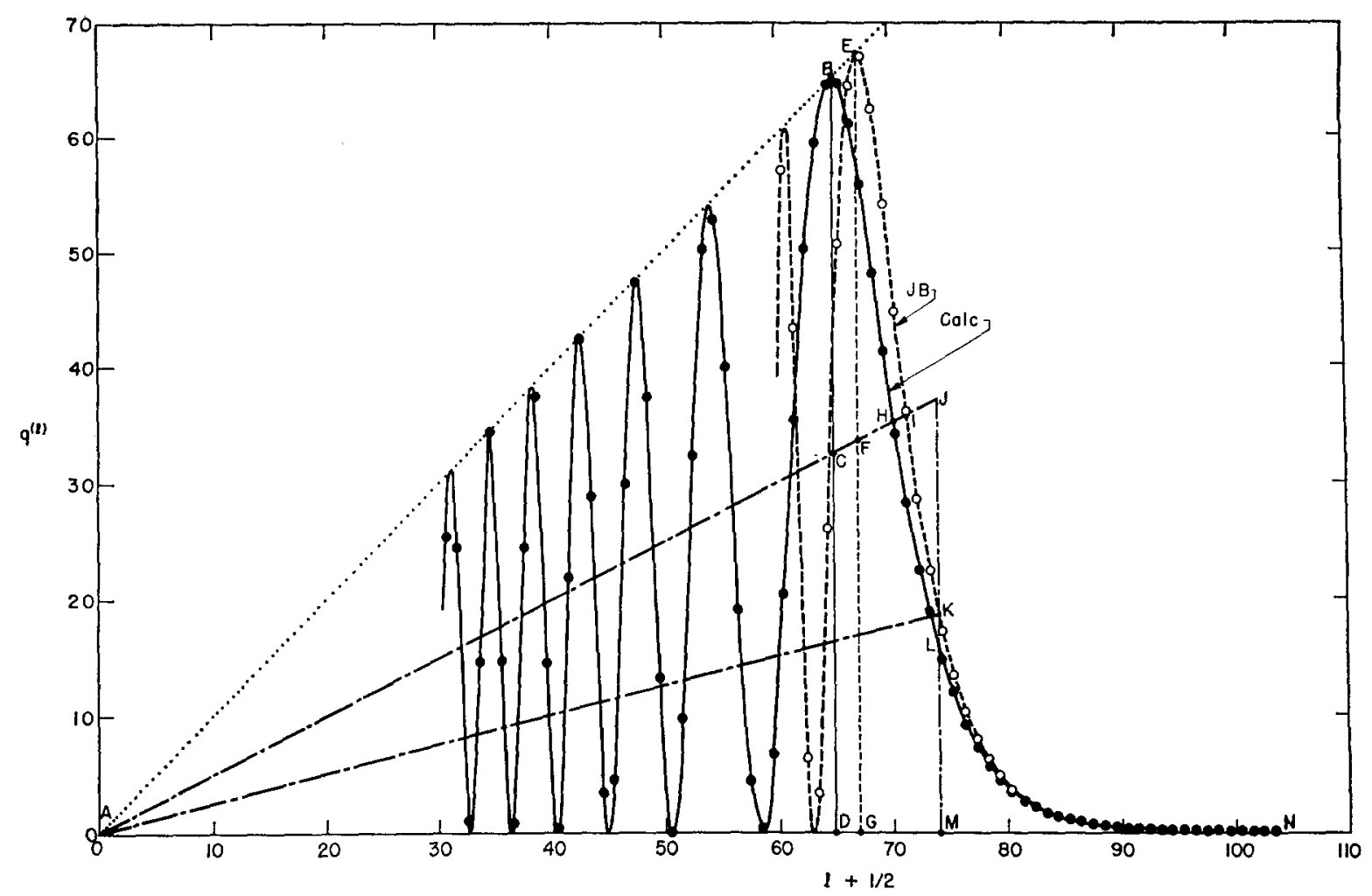

FIG. 2. Graphical presentation of partial cross sections: $q \ldots$ vs $l+\frac{1}{2}$, for $s=12$ repulsion. Solid circles: Partial wave; open circles: JB approximation. For clarity, values for low $l$ are not shown on the graph.

so that the contribution to the integrals from $y_{0}>1.5$ is obtainable in closed form. In this way the phases $\eta_{l}(69.282)$ were evaluated for $0 \leq l \leq 102$. For comparison, higher-order phases $(l \geq 50)$ were calculated by the JB approximation:

$$
\left(\eta_{l}\right)_{\mathrm{JB}}=-\frac{63}{256} \pi\left[B A^{10} /\left(l+\frac{1}{2}\right)^{11}\right] .
$$

for $l \geq 78$ (for which $\eta_{l}=-0.27$ ), the JB phases agreed within \pm 0.01 with the semiclassically calculated phases. A few of the low-order phases (e.g., $\eta_{0} \cong-59$ rads) were checked (approximately) by the "exact" method" involving integration of the radial wave equation.

The approximation formulas for the cross section [Eq. (2)], expressed in the reduced notation [see Eq. (13)], are:

$$
\left.Q_{\mathrm{MLL}}^{*{ }_{\mathrm{MM}}}={ }_{2.377}^{2.273}\right\} \cdot\left(\frac{B}{A}\right)^{2 / 11} .
$$

Table II lists the numerical results. These approximate values should be compared with the "true" (partialwave calculated) value, $Q_{0}{ }^{*}$. Unfortunately, the calculated $Q_{0}{ }^{*}$ was found to be quite sensitive to the accuracy of the higher-order phases, so that a range of values was obtained, depending upon different round-off procedures, etc. The "best" value (with the probable error indicated) is listed; it is not significantly different from the SLL result.

To extrapolate from this one example would be haz- ardous; however, a graphical presentation makes it possible to visualize the difference in the three procedures (yielding $Q_{\mathrm{MM}}{ }^{*}, Q_{\mathrm{SLL}}{ }^{*}$, and $Q_{0}{ }^{*}$, respectively), and makes plausible the generality of this conclusion.

Figure 2 shows a graph of "partial" cross sections $q^{(l)}\left[\right.$ see Eq. (13)] vs $l+\frac{1}{2}$. The dotted line $(A E)$ of slope unity represents the upper bound on $q^{(l)}$. The area under the oscillating solid curve (passing through its final maximum at $B$ and then decreasing monotonically along $B H L N$ ), when multiplied by $8 / A^{2}$, yields a "graphical" value of $Q_{0}{ }^{*}$, listed in Table II, in good agreement with the computed "true" cross section; the area under the corresponding JB curve yields $Q_{\mathrm{SLL}}{ }^{*}$. The $Q_{\mathrm{M} \mathrm{M}}{ }^{*}$ value is derived from the sum of areas $A J M$ and $M K N$; the line $A J$ is of slope $\frac{1}{2}=\left\langle\sin ^{2} \eta_{l}\right\rangle_{\mathrm{a}} v$. Point $M$ has been located by the intersection of line $A K$, of

TABLE II. Specific results of various approximations, for the $s=12$ example.

\begin{tabular}{llll}
\hline & Numerical & Graphical & $\begin{array}{l}\text { Simplified } \\
\text { Graphical }\end{array}$ \\
\hline$Q_{0}{ }^{*}$ & $2.54_{6} \pm 0.01$ & $2.54 \pm 0.02$ & $2.50 \pm 0.02$ \\
$Q_{\mathrm{SLL}^{*}}$ & 2.541 & $2.54 \pm 0.02$ & $2.50 \pm 0.02$ \\
$Q_{\mathrm{MM}}{ }^{*}$ & 2.430 & $2.41_{8} \pm 0.01$ & $2.41_{2} \pm 0.02^{\mathrm{a}}$ \\
\hline
\end{tabular}

A Using Eq. (26) from reference 13: $Q_{M M}{ }^{*}=(21 / 10) \beta_{L}^{2}$ [where $\beta_{L}=$ $\left(L+\frac{1}{2}\right) / A$ ], and locating point $M$ from Fig. 2 . 
slope $=\frac{1}{4}$ (i.e., $\left.\sin \eta_{\mathrm{JB}} \cong \eta_{\mathrm{JB}}=\frac{1}{2}\right)$, with the JB curve $(E I N)$. The graphical result (listed) agrees well with the calculated value.

As a simplified approximation, $Q_{0}{ }^{*}$ may be estimated by summing areas $A C D+D B N(=A C B N)$, yielding the value designated "simplified graphical". Similarly, $Q_{\mathrm{SLL}}{ }^{*}$ may be approximated by the sum $A F G+G E N$ (=AFEN); the same value is obtained. This good agreement is obviously due to a compensation of errors in the SLL approximation: the "true" area $D B N$ is well approximated by the sum of areas $D C F G+G E N$ $(=D C F E N)$.

The difference between $Q_{\mathrm{SLL}} *$ and $Q_{\mathrm{MM}} *$ is accounted for almost entirely by the difference between areas $F E I$ and $I J K$ (also, compare $C B H$ with $H J L$ ).

\section{CONCLUDING REMARKS}

The preceding sections have shown the equivalence of the S and LL approximations and their relationship to that of MM. Since all can be derived from the same assumption (the classical low-angle deflection function) via the semiclassical equivalence relationship, the same dependence of $Q$ upon $C$ and $v$ must follow. From the numerical-graphical illustration presented (for $s=12$ ) it appears that the SLL approximation is superior to the $\mathrm{MM}$ formula, $Q_{\mathrm{sLL}}{ }^{*}$ agreeing (within an uncertainty of $\pm \frac{1}{2} \%$ ) with the "true" value, $Q_{0}{ }^{*}$. It is seen that the principal source of the bias in the MM formulation is neither the error in the JB phases (for $l \geq L$ ) nor the approximation $\sin \eta_{\mathrm{JB}} \cong \eta_{\mathrm{JB}}\left(\right.$ for $\left.\eta_{l}<\frac{1}{2}\right)$, but rather the "nonrandom" phases in the important region of $l$ just below $L$, i.e., where $\pi / 2 \geq \eta_{l} \geq \frac{1}{2}$.

The evidence on the basis of calculations for the $\mathrm{L}-\mathrm{J}$ $(12,6)$ potential has also been reviewed, and it is inferred that a velocity-averaged "true" cross section would differ from the SLL approximation $Q_{\mathrm{SLL}}{ }^{(6)}$ by less than $\pm 1.5 \%$.

The following procedure for analysis of cross-section data is therefore recommended. A plot of $\log Q$ vs $\log v$ is made, inspection indicating the velocity range over which the mean curve (averaging out the undulations) has a slope of $-2 / 5$. Over this range a plot should be made of the "apparent" value of the potential constant $C_{\text {app }}{ }^{(6)}$, calculated from the SLL formula:

$$
\left.C_{\text {app }}{ }^{(6)}(\mathrm{erg} \mathrm{cm})^{6}\right)=5.676 \times 10^{-30} v Q^{5 / 2},
$$

as a function of $v^{-1}$ (in such a plot the extrema are approximately evenly spaced ${ }^{12}$ ). The average value of $C_{\text {app }}{ }^{(6)}$ may be taken as the true value of $C^{(6)}$. For measurements with Maxwellian beams, the velocityaveraging has already been effectively accomplished, so that the influence of the repulsion has been largely removed; thus the value of $C$ calculated by Eq. (18) should be close to the true value.

\section{ACKNOWLEDGMENTS}

The authors thank Mr. F. Parker and Mr. A. Flank for their help in the calculations, and acknowledge helpful suggestions from Dr. E. W. Rothe and Professor E. A. Mason. 